\title{
Lactobacillus GG eine Option bei blutigen Stühlen im Säuglingsalter
}

In einer aktuellen Studie wurde bei Säuglingen mit Hämatochezie zum einen der diagnostische Wert von Calprotectin im Stuhl als fäkaler Inflammationsmarker untersucht. Zum anderen wurde das Ansprechen auf eine kuhmilchfreie Ernährung (extensives Hydrolysat) mit und ohne Zugabe von Lactobacillus GG geprüft.

lutige Stühle bei jungen Säuglingen, entweder unter Muttermilchnahrung oder unter einer Kuhmilch-Formulaernährung, stellen ein häufiges Problem in der Kinderarztpraxis dar. Die Standardtherapie besteht darin, bei fehlender spontaner Besserung innerhalb von 1-2 Wochen einen Versuch mit einer kuhmilchproteinfreien Ernährung zu unternehmen. Ein Problem stellt die Tatsache dar, dass der Verlauf einer Kolitis nicht invasiv z.T. schwierig zu beurteilen ist. In der aktuellen Studie wurde einerseits der diagnostische Wert von Calprotectin im Stuhl als Verlaufsparameter, andererseits das Ansprechen auf eine kuhmilchfreie Ernährung (extensives Hydrolysat) mit und ohne Zugabe von Lactobacillus GG (LGG) untersucht. Endoskopische Untersuchungen wurden nicht durchgeführt. Beteiligt waren 30 Patienten mit blutigen Stühlen und 32 Gesunde als Kontrollgruppe.

Bei den Patienten mit blutigen Stühlen fand sich mit $325 \mu \mathrm{g} / \mathrm{g}$ Stuhl (Range: 269-388) ein signifikant höherer Calprotectingehalt als in der gesunden Kontrollgruppe mit $132 \mu \mathrm{g} / \mathrm{g}$ Stuhl (Range: 119-145). Der Pricktest mit Milch war bei $10 \%$ der Patienten mit blutigen Stühlen positiv, der Patchtest mit Milch bei einem von 30 Patienten.

Die formulaernährten Kinder mit blutigen Stühlen wurden dann randomisiert und bekamen entweder eine Hydrolysatmilch mit oder ohne LGG. Das Ausmaß des Rückgangs des Calprotectins war abhängig davon, welche Nahrung die Kinder bekommen hatten: Der Abfall des fäkalen Calprotectins war signifikant höher bei Patienten, die zur Hydrolysatnahrung noch LGG bekamen. Bei den Kindern, denen Hydrolysat ohne LGG gefüttert wurde, war der Rückgang des Calprotectins vergleichbar dem physiologischen Verlauf des Calprotectins in der gesunden Kontrollgruppe.
Kommentar: Die vorliegende Studie unterstützt die Hypothese, dass nicht nur allergische Mechanismen für blutige Stühle im Säuglingsalter bzw. die Säuglingskolitis verantwortlich sind, sondern auch Störungen der fäkalen Mikroflora, die bisher nicht bewiesen oder quantifiziert werden konnten. Bekannt ist ja, dass die Mikroflora des Dickdarms eine wichtige Rolle auch für die Ernährung der Dickdarmmukosazellen hat, dass auch bei anderen entzündlichen Darmerkrankungen Störungen der Mikroflora vorkommen und dass Probiotika bei vielen Kolitisformen therapeutische Effekte haben können. In diesen Zusammenhang sind die positiven Effekte von LGG einzuordnen.

Auch wenn die Zahl der untersuchten Kinder gering war und diese Untersuchung sicher durch eine zweite Arbeitsgruppe kontrolliert und bestätigt werden sollte, erscheint ein Versuch mit der Gabe von LGG über vier Wochen bei Kindern mit blutigen Stühlen im jungen Säuglingsalter gerechtfertigt.

Dr. Martin Claßen

Baldassarre ME et al. Lactobacillus GG improves recovery in infants with blood in the stools and presumptive allergic colitis compared with extensively hydrolyzed formular alone. J Pediatr $2010 ; 156: 397-401$

\section{Mumpsorchitis nimmt bedenklich zu}

\section{Ärzte eines Krankenhauses in Dublin beobachteten in den letzten Jahren einen steilen Anstieg der Mumpsorchitis bei 15 bis 24 Jahre alten Jugend- lichen. Sie nahmen diese Fälle genauer unter die Lupe.}

Ä rzte aus Irland werteten die Akten von Patienten mit urologischen Krankheiten an ihrem Krankenhaus in Hinblick auf eine Mumpserkrankung aus. Hauptergebnisse waren: Rund $42 \%$ der Mumpsinfizierten bekommen mindestens eine Komplikation. Beim Mann ist es meistens die Mumpsorchitis. Fakten der Untersuchungen waren: Bis zu 50\% der Männer mit Mumpsorchitis bekommen eine Hodenatrophie, wobei ein oder beide Testikel verkleinert sein können. Die totale Infertilität ist selten. Aber eine Subfertilität kann bei bis zu 13\% der Patienten vorliegen, auch wenn beide Testikel nicht geschrumpft sind.

Bis zu 50\% der Patienten haben bis zu drei Monate nach der Entzündung anomale Spermien. 24\% der Erwachsenen und $38 \%$ der Heranwachsenden haben diese Veränderungen noch drei Jahre später. Es scheint eine direkte Beziehung zwischen der Testikelschwellung und der Zahl der Spermienanomalien zu bestehen. Die Assoziation zwischen reduzierter Testikelgröße und Hodenkarzinomrisiko ist mit einer Inzidenz von 0,5\% schwach.
Kommentar: Der Bericht der irischen Wissenschaftler ist auch für Deutschland bedeutsam. Die Ursache der meisten Mumpserkrankungen ist die Ablehnung der MMR-Impfung der Kinder durch die Eltern. In den 1990er-Jahren wurde in der Presse über einen Zusammenhang zwischen Autismus und der MMR-Impfung spekuliert. Die Durchimpfungsrate fiel daraufhin vielerorts von $91 \%$ auf $58 \% \mathrm{ab}$.

Wir Ärzte werden wieder häufiger Patienten mit Mumpskomplikationen in der Praxis haben. Wir sollten uns daher mit diesen Komplikationen vertraut machen und alles unternehmen, um Eltern zu überzeugen, dass die MMR-Impfung die wirksamste und kostengünstigste Maßnahme ist, um die Kinder vor den Risiken und Komplikationen einer Mumpsinfektion zu schützen.

Prof. Dr. Kurt Malberg

Davis NF et al. The increasing incidence of mumps orchitis: a comprehensive review. BJU Int 2010; 105: 1060-5 